\title{
Salivary CXCL13 in relation to scintigraphy in early detection of secondary Sjogren's syndrome
}

Salwa G. Moussa ${ }^{a}$, Hanan E. El-Hefnawy ${ }^{a}$, Heba F. El-Shishtawy ${ }^{a}$, Dalia M.E. El Mikkawy ${ }^{a}$, Mennatallah H. Shalaby ${ }^{b}$

Departments of aPhysical Medicine, Rheumatology and Rehabilitation, ${ }^{\text {b Radiology, }}$ Faculty of Medicine, Ain Shams University, Cairo, Egypt

Correspondence to Dalia M.E. El-Mikkawy, MD of Physical Medicine, Rheumatology and Rehabilitation, Physical Medicine,

Rheumatology and Rehabilitation Department, Faculty of Medicine, Ain Shams University, Cairo, Egypt. Tel: +20 122580 0199/+20 102 558 5779;

e-mail: drdaliaezz74@yahoo.com

Received 24 July 2018

Accepted 16 August 2018

Egyptian Rheumatology \& Rehabilitation 2018, 45:153-158

\section{Background}

Sjogren's syndrome (SS) is a systemic autoimmune disease in which immune cells attack and destroy the exocrine glands. CXCL13 directs B-cell chemotaxis and is elevated in several autoimmune diseases.

Objective

To assess the role of salivary CXCL13 level as a screening tool in early detection of secondary SS patients.

Patients and methods

Salivary CXCL13 levels using ELISA technique, Schirmer paper test and/or Lissamine green staining, and quantitative salivary scintigraphy excretion fraction were measured in 45 selected patients with primitive connective tissue disease (rheumatoid arthritis, systemic lupus erythematosus, or systemic sclerosis) and according to the American-European Consensus Group criteria, they were classified to three equal groups: group I were having SS; group II were having dryness manifestations but not completing the criteria for SS diagnosis (suspected SS); group III were having no SS, and 15 age-matched and sex-matched apparently healthy controls.

\section{Results}

A significantly higher salivary CXCL13 level on comparing SS patients to suspected, non-SS groups and controls $(P<0.001)$. Salivary CXCL13 had a significant negative correlation with scintigraphy $(P<0.01)$, a significant positive correlation with eye dryness signs $(P<0.01)$, cutoff value of CXCL13 to diagnose SS was more than $40 \mathrm{pg} / \mathrm{ml}$ and a cutoff value of salivary scintigraphy excretion fraction to diagnose SS was less than $33.1 \%$.

\section{Conclusion}

Salivary CXCL13 is a sensitive biomarker for early detection of secondary SS.

\section{Keywords:}

CXCL13, salivary, scintigraphy, Sjogren', s syndrome

Egypt Rheumatol Rehabil 45:153-158

(C) 2018 Egyptian Society for Rheumatology and Rehabilitation $1110-161 X$

\section{Introduction}

Sjogren's syndrome (SS) is a systemic autoimmune disease in which immune cells attack and destroy the exocrine glands that produce tears and saliva [1].

B cells have been implicated in the pathogenesis of SS, as they secrete inflammatory cytokines and produce autoantibodies that focus inflammation. Significantly, B cells also influence the differentiation of T-helper cells, specifically Th17 and T regulatory subsets, which are implicated in the modulation of autoimmune disease [2].

It has been suggested that chronic inflammation of exocrine glands depends on chemokines, which control the selective traffic and tissue homing of inflammatory cells [3].

CXCL13 directs B-cell chemotaxis and is elevated in several autoimmune diseases. A study demonstrates that in humans, CXCL13 is elevated in the serum and saliva, and an elevated salivary CXCL13 level distinguishes patients with xerostomia $[4,5]$. These data suggest a role for CXCL13 as a valuable biomarker in SS, as 74\% of patients with SS displayed elevated CXCL13 in the sera, saliva, or both [5]. Thus, CXCL13 may be pathogenically involved in SS and may serve as a new marker and as a potential therapeutic target.

Salivary scintigraphy is a noninvasive method to evaluate the function of salivary glands by addressing the uptake and secretion of a radioactive-labelled substance (sodium pertechnate of ${ }^{99} \mathrm{~m} \mathrm{Tc}$ ). Additionally, an abnormal salivary gland scintigraphy result is accepted by the

This is an open access journal, and articles are distributed under the terms of the Creative Commons Attribution-NonCommercial-ShareAlike 4.0 License, which allows others to remix, tweak, and build upon the work non-commercially, as long as appropriate credit is given and the new creations are licensed under the identical terms. 
American-European Consensus Group as a criterion for the diagnosis of SS [6].

\section{Patients and methods}

The present study is a case-control one that included 45 patients having either rheumatoid arthritis, systemic lupus erythematosus, or systemic sclerosis. They were selected from inpatients and outpatients in the Ain Shams University Hospitals. Fifteen age-matched and sex-matched apparently healthy individuals were also included in the study and served as a control group. Written consent was obtained from all patients and controls after a full explanation of the study was provided. Ethics committee approval was obtained for the study.

The selected patients were divided into three equal groups as follows: patients with SS according to the American-European Consensus Group criteria [7], patients suspicious to have SS (not fulfilling the criteria), and patients with no manifestations of SS.

\section{Exclusion criteria}

Past head and neck radiation treatment, use of anticholinergic drugs, facial paralysis, diabetes mellitus, multiple sclerosis, graft-versus-host disease, sarcoidosis, hepatitis $\mathrm{C}$ virus, and pregnant ladies.

All patients were subjected to laboratory investigations such as salivary CXCL13 levels using ELISA technique, complete blood picture, erythrocyte sedimentation rate, and $\mathrm{C}$-reactive protein.

For dry eye diagnosis Lissamine green (LG) staining and/or Schirmer paper test were done (Figs 1 and 2).

For dry mouth diagnosis quantitative salivary gland scintigraphy to evaluate the function of salivary glands

\section{Figure 1}

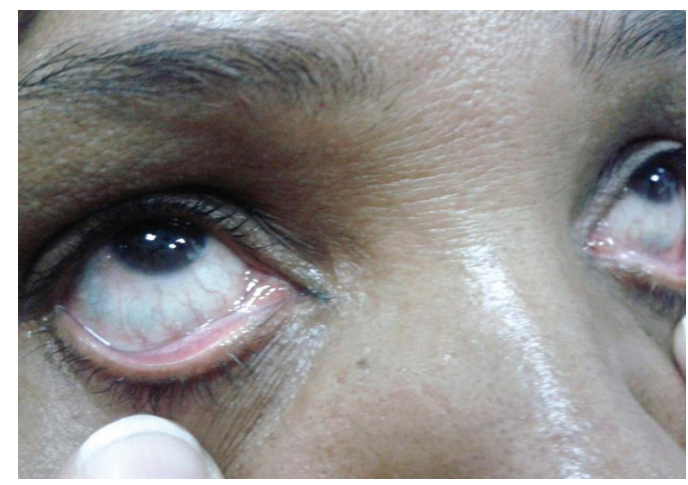

Lissamine green temporal stain (moderate degree) in right. Eye of a rheumatoid arthritis patient with secondary Sjogren's syndrome. by addressing the uptake and secretion of a radioactivelabelled substance (sodium pertechnate of ${ }^{99 \mathrm{~m}} \mathrm{Tc}$ ) and then calculate the excretion fraction (EF) (Fig. 3).

Statistical presentation and analysis was carried out by IBM SPSS statistics (version 17.0, 2009; IBM Corp., Chicago, USA) used for data analysis. The mean and $\mathrm{SD}$, the medians, Student $t$-test, $\chi^{2}$, Kruskal-Wallis test, and analysis of variance tests were used to summarize the characteristics of the samples. Categorical variables were summarized with frequencies and percentages. Spearman's correlation coefficients were established with continual values of salivary CXCL13, salivary gland scintigraphy $\mathrm{EF}$, and eye dryness signs, depending on the distribution of the variables. Receiver operating characteristic (ROC) curves were calculated to establish sensitivity, specificity for CXCL13 salivary level, and scintigraphy EF. A value of $P$ less than 0.05 was considered significant.

\section{Figure 2}

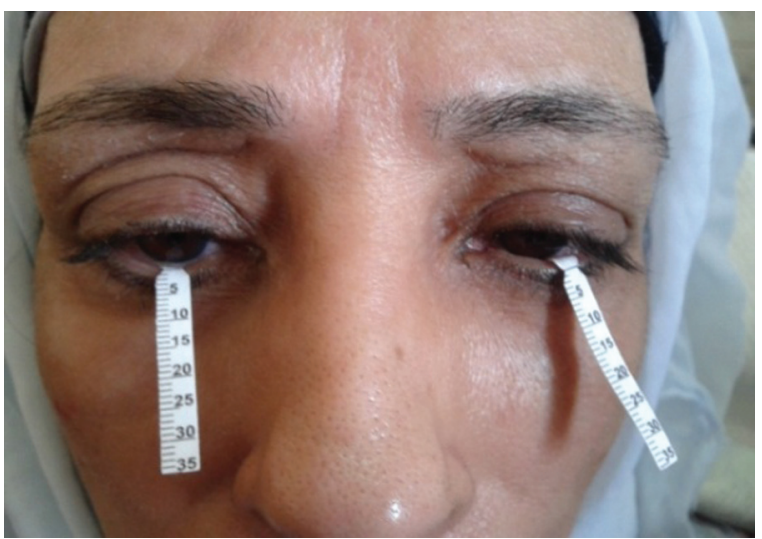

Schirmer paper test showing positive results in both patients $(<5 \mathrm{~mm}$ in $5 \mathrm{~min}$ ).

Figure 3

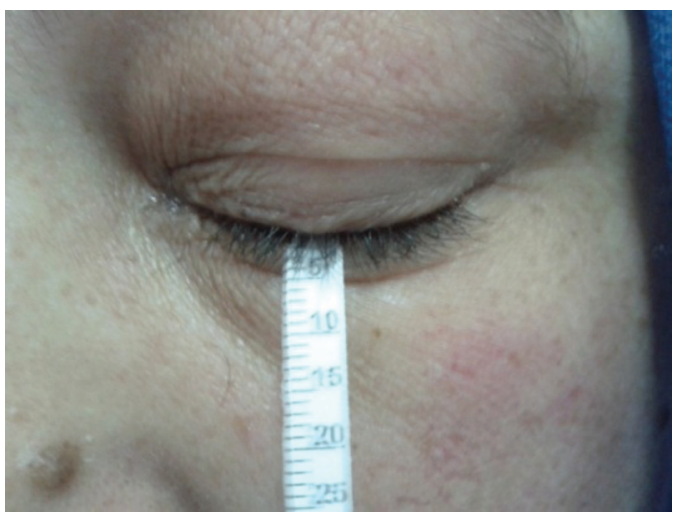

Quantitative salivary gland scintigraphy of an Sjogren's syndrome patient before applying lemon juice at $10-12 \mathrm{~min}$ postinjection, after applying lemon juice at 18-20 min postinjection. Region of interest (ROIs) used for quantification are depicted on the left scintigram. 


\section{Results}

This study included 45 patients with primitive connective tissue disease (rheumatoid arthritis, systemic lupus erythematosus, or systemic sclerosis), classified according to the American-European Consensus Group criteria for SS diagnosis into three equal subgroups: group I, group II, and group III.

Group I included 15 patients with SS, 15 (100\%) women and zero $(0.0 \%)$ men; their ages ranged from 30.0 to 58.0 years with a median of 41 years. Their dryness manifestations duration ranged from 0.58 to 10.0 years, with a median of 2 years.

The salivary CXCL13 level ranged from 44 to $365 \mathrm{pg} /$ $\mathrm{ml}($ mean \pm SD 147.87 \pm 97.74$)$.

The salivary gland scintigraphy $\mathrm{EF}(\%)$ of the right $(\mathrm{Rt})$ parotid gland (mean \pm SD $22.58 \pm 10.8$ ), left $(\mathrm{Lt}$ ) parotid gland (mean \pm SD $24.58 \pm 7.12$ ), Rt submandibular gland (mean \pm SD 30.39 \pm 8.85$)$, and Lt submandibular gland $($ mean \pm SD 20.58 \pm 7.55$)$.

Schirmer paper test and/or LG stain were positive in 15 (100\%) patients.

Group II included 15 patients with suspected SS (having dryness manifestations but not completing criteria of SS diagnosis), 14 (93.3\%) women and one (6.7\%) man; their ages ranged from 15 to 60 years with a median of 50 years. Their dryness manifestations duration ranged from 0.08 to 15 years, with a median of 4 years.

The salivary CXCL13 level ranged from 11 to $142 \mathrm{pg} /$ $\mathrm{ml}($ mean $\pm \mathrm{SD} 46.9 \pm 35.4)$.
The salivary gland scintigraphy EF (\%) of the Rt parotid gland (mean \pm SD $34.05 \pm 6.84)$, Lt parotid gland (mean \pm SD $35.17 \pm 8.47$ ), Rt submandibular gland (mean \pm SD 19.31 \pm 8.68 ), and Lt submandibular gland (mean \pm SD $33.59 \pm 9.73)$.

Schirmer paper test and/or LG stain were positive in nine $(60.0 \%)$ patients.

Group III included 15 patients with no SS (not suspected nor having SS), 14 women (93.3\%) and one $(6.7 \%) \mathrm{man}$; their ages ranged from 17 to 58 years with a median of 41 years.

The salivary CXCL13 level ranged from 10 to $34 \mathrm{pg} / \mathrm{ml}$ (mean \pm SD 23.57 \pm 8.63 ).

The salivary gland scintigraphy EF (\%) of the Rt parotid gland (mean \pm SD 39.39 \pm 9.3 ), Lt parotid gland (mean \pm SD $37.11 \pm 6.4$ ), Rt submandibular gland (mean \pm SD $34.13 \pm 5.59$ ), and Lt submandibular gland (mean \pm SD $35.40 \pm 6.09$ ).

Schirmer paper test and/or LG stain were positive in zero $(0.0 \%)$ patient.

The clinical, laboratory, and scintigraphy data of the patients are shown in Tables $1-3$.

There was a highly significant increase in salivary CXCL13 in SS patients versus the suspected SS, non-SS, and control groups $(P<0.001)$. There was a significant increase in salivary CXCL13 in suspected SS group versus the control group $(P=0.031)$. There was a statistically significant positive relation between

Table 1 Frequencies of ocular dryness manifestations among patient groups

\begin{tabular}{|c|c|c|c|c|c|c|}
\hline \multirow[t]{2}{*}{ Ocular dryness manifestations } & \multicolumn{4}{|c|}{ Groups $[n(\%)]$} & \multirow[t]{2}{*}{$\chi^{2}$} & \multirow[t]{2}{*}{$P$-value } \\
\hline & SS group & Suspected group & No SS group & Total & & \\
\hline \multicolumn{7}{|l|}{ LG or SP test } \\
\hline Negative & $0(0.00)$ & $6(40.00)$ & $15(100.00)$ & $21(46.67)$ & 30.536 & $<0.001^{*}$ \\
\hline Positive & $15(100.00)$ & $9(60.00)$ & $0(0.00)$ & $24(53.33)$ & & \\
\hline \multicolumn{7}{|l|}{ Dry for 3 months } \\
\hline Negative & $1(6.67)$ & $8(53.33)$ & $15(100.00)$ & $24(53.33)$ & 26.250 & $<0.001^{*}$ \\
\hline Positive & 14 (93.33) & $7(46.67)$ & $0(0.00)$ & $21(46.67)$ & & \\
\hline \multicolumn{7}{|l|}{ Sand sensation } \\
\hline Negative & $6(40.00)$ & $8(53.33)$ & $15(100.00)$ & $29(64.44)$ & 12.996 & $0.002^{*}$ \\
\hline Positive & $9(60.00)$ & $7(46.67)$ & $0(0.00)$ & $16(35.56)$ & & \\
\hline \multicolumn{7}{|l|}{ Artificial tears three times/day } \\
\hline Negative & $9(60.00)$ & $6(40.00)$ & $15(100.00)$ & $30(66.67)$ & 12.600 & $0.002^{*}$ \\
\hline Positive & $6(40.00)$ & $9(60.00)$ & $0(0.00)$ & $15(33.33)$ & & \\
\hline Total of positive eye manifestations & $15(100 \%)$ & $13(86.7)$ & $0(0.00)$ & $28(62.2)$ & 37.6 & $<0.001^{*}$ \\
\hline
\end{tabular}

It shows a highly statistically significant difference between patient groups as regards dryness manifestations (SS $>$ suspected $S S>$ nonSS). LG, Lissamine green; SP, Schirmer paper test; SS, Sjogren's syndrome. *Means significant difference. 
Table 2 Comparison between patients and control groups as regards salivary CXCL13 levels

\begin{tabular}{|c|c|c|c|c|c|c|c|}
\hline \multirow[t]{2}{*}{ Groups } & \multicolumn{5}{|c|}{ CXCL13 saliva (pg/ml) } & \multicolumn{2}{|c|}{ Kruskal-Wallis test } \\
\hline & \multicolumn{2}{|c|}{ Range } & Median & 10 & & $H$ & $P$-value \\
\hline SS group & \multicolumn{2}{|c|}{$44-365$} & 134.00 & $17 \varepsilon$ & .00 & 32.27 & $<0.001^{*}$ \\
\hline Suspected SS group & \multicolumn{2}{|c|}{$11-142$} & 34.00 & 44 & & & \\
\hline No SS group & \multicolumn{2}{|c|}{$10-34$} & 24.00 & 17 & 50 & & \\
\hline Control group & \multicolumn{2}{|c|}{$0.1-39.1$} & 27.00 & 24 & & & \\
\hline \multicolumn{8}{|c|}{ Mann-Whitney test } \\
\hline SS and suspected SS & SS and non-SS & SS and control & Suspecte & & Sus! & d control & Non-SS and control \\
\hline $0.001^{*}$ & $<0.001^{*}$ & $<0.001^{*}$ & & & & & 0.95 \\
\hline
\end{tabular}

It shows a statistically significant difference between SS versus suspected SS, SS versus non-SS, SS versus control, and suspected SS versus control. SS, Sjogren's syndrome. *Means significant difference.

Table 3 Comparison between patients and control group as regards salivary gland scintigraphy (excretion fraction) of the right parotid gland

\begin{tabular}{|c|c|c|c|c|c|}
\hline \multirow[t]{2}{*}{ Groups } & & \multicolumn{2}{|c|}{$\begin{array}{l}\text { Salivary scintigraphy of right parotid gland } \\
(\text { EF\%) }\end{array}$} & \multirow[t]{2}{*}{$F$} & \multirow[t]{2}{*}{$P$-value } \\
\hline & & Range & Mean \pm SD & & \\
\hline SS group & & $7.6-49$ & $22.58 \pm 10.81$ & 11.11 & $<0.001^{*}$ \\
\hline Suspected group & & $25.2-47$ & $34.05 \pm 6.84$ & & \\
\hline No SS group & & $25.9-55.6$ & $39.39 \pm 9.29$ & & \\
\hline Control group & & $26.7-59.4$ & $38.27 \pm 8.28$ & & \\
\hline \multicolumn{6}{|c|}{ Tukey's test } \\
\hline SS and suspected & SS and NSS & SS and control & Suspected and NSS & Suspected and control & NSS and control \\
\hline $0.005^{*}$ & $<0.001^{*}$ & $<0.001^{*}$ & 0.37 & 0.57 & 0.99 \\
\hline
\end{tabular}

It shows a statistically significant difference between SS versus suspected SS, SS versus non-SS, and SS versus control. EF, excretion fraction; SS, Sjogren's syndrome. *Means significant difference.

Table 4 Correlation between levels of salivary CXCL13 and salivary glands scintigraphy excretion fraction

\begin{tabular}{lcc}
\hline Salivary gland scintigraphy (EF\%) & \multicolumn{2}{c}{ CXCL13 saliva } \\
\cline { 2 - 3 } & $r$ & $P$-value \\
\hline Salivary scintigraphy right parotid & -0.610 & $<0.001^{*}$ \\
Salivary scintigraphy left parotid & -0.629 & $<0.001^{*}$ \\
Salivary scintigraphy right submandibular & -0.679 & $<0.001^{*}$ \\
Salivary scintigraphy left submandibular & -0.671 & $<0.001^{*}$ \\
\hline
\end{tabular}

It shows a negative statistically significant correlation. EF, excretion fraction. *Means significant difference.

salivary CXCL13 levels and positive eye staining and Schirmer paper test.

There was a significant negative correlation between salivary CXCL13 levels and salivary gland scintigraphy EF $\quad(r=0.65, \quad P<0.001) \quad($ Table 4$)$. There was insignificant relation between salivary CXCL13 levels and either sex, age, or duration of SS $(P>0.05)$. Using salivary CXCL13 levels above $40 \mathrm{pg} / \mathrm{ml}$ as a cutoff point, the ROC curve analysis showed a sensitivity of $100 \%$ and a specificity of $60 \%$, with an area under the curve of 0.871 (95\% confidence interval: 0.698-0.965) (Fig. 4).

There was a statistically significant difference between salivary gland scintigraphy EF of SS group and other groups $(P<0.001)$. There was no significant correlation between salivary gland scintigraphy and duration of SS $(r=0.292, P>0.05)$. The salivary gland scintigraphy EF cutoff value was less than $33.1 \%$ with a sensitivity of $100 \%$ and a specificity of $86.7 \%$ was used in the diagnosis of oral dryness in SS (Fig. 5).

\section{Discussion}

Sjogren's syndrome is a systemic autoimmune disease in which immune cells attack and destroy the exocrine glands that produce tears and saliva [1]. The discovery of novel markers and diagnostic methods is crucial for the diagnosis and management of SS, as a disease stage criteria are lacking, and there is no standardized way to monitor SS progression [8].

In our study comparison between patients and controls regarding salivary CXCL13 revealed a highly significant increase $(P<0.001)$ in salivary CXCL13 in patients compared with controls (134 vs. $27 \mathrm{pg} /$ $\mathrm{ml}$ ), respectively, suggesting a pathogenic link between CXCL13 and SS, this is in agreement with Kramer et al. [8], a statistically significant difference between SS group versus suspected SS, also between SS 


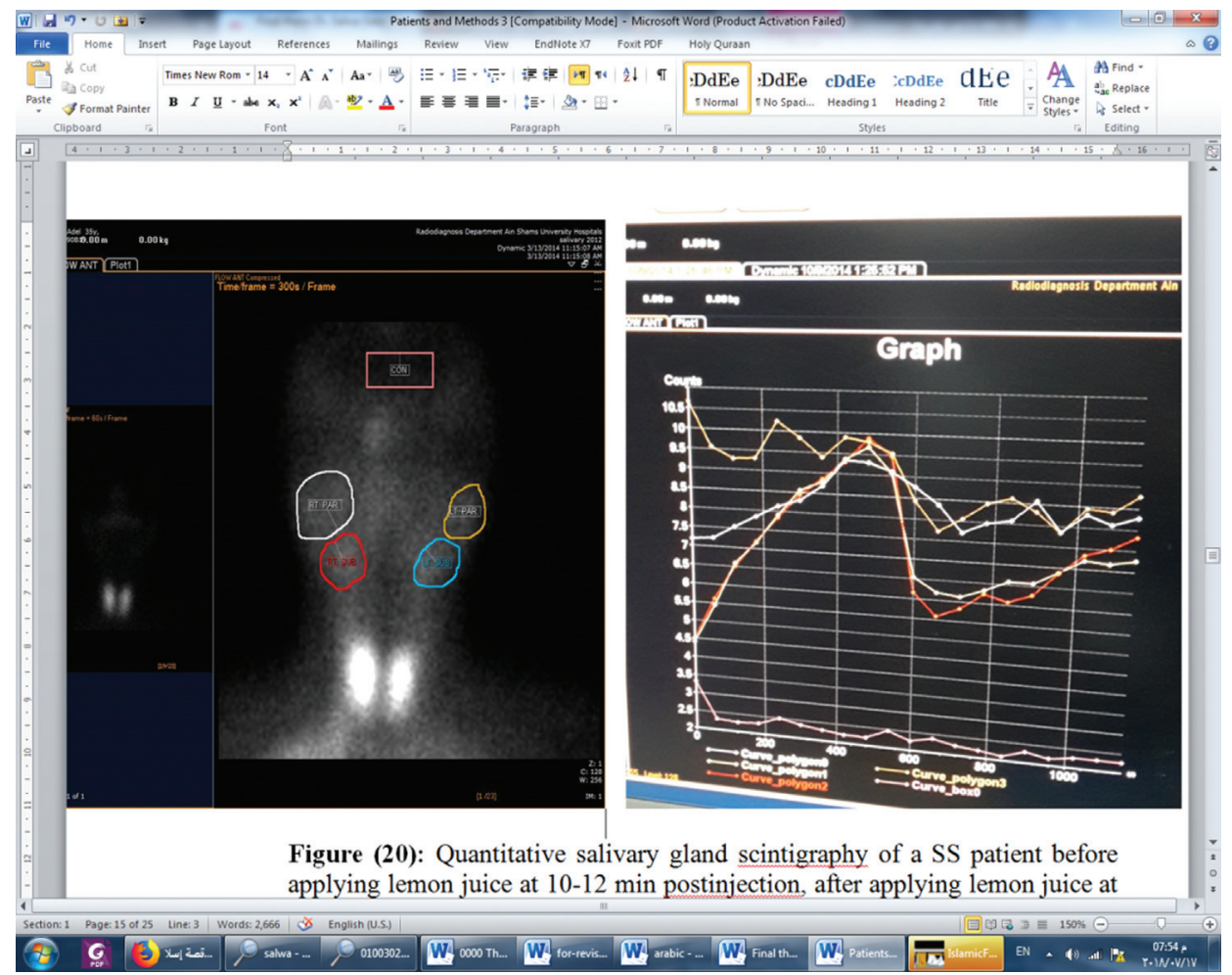

Receiver operating characteristic (ROC) curve for CXCL13 salivary levels

Figure 5

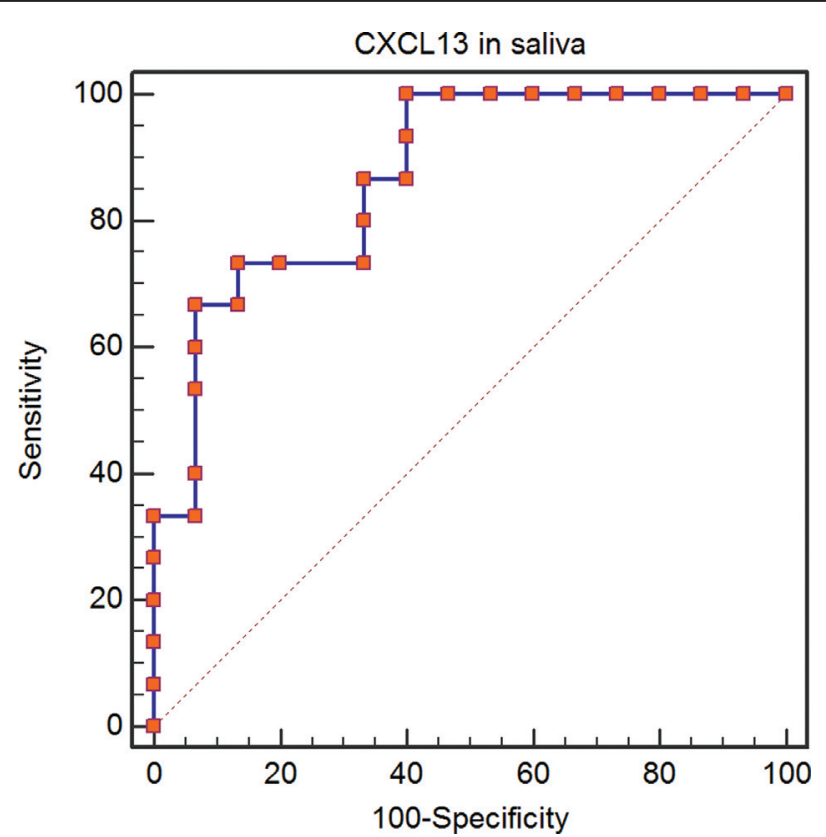

Receiver operating characteristic (ROC) curve for salivary gland scintigraphy.

group versus NSS group and SS group versus control group, and suspected SS group versus control group $(P<0.001)$. The difference was not statistically significant between suspected group versus non-SS group, and non-SS versus the control $(P>0.05)$. So
CXCL13 salivary levels increased markedly in patients with SS and to a little extent in suspected patients.

As B cells have been implicated in the pathogenesis of SS [2], and CXCL13 directs B-cell chemotaxis [4], this might explain the rising of salivary CXCL13 in SS patients.

The observation of Lee et al. [9] that the expression levels of CXCL13 within the lymphocytic infiltrates of SS patients was associated with several laboratory features of the disease, lymphadenopathy, and the extent of clinical disease activity recommends CXCL13 as a useful marker for predicting SS disease activity and prognosis.

The cutoff value of salivary CXCL13 to diagnose SS was $40 \mathrm{pg} / \mathrm{ml}$, with a sensitivity of $100 \%$ and specificity of $60 \%$, while in Kramer et al. [8], the cutoff value was $100.3 \mathrm{pg} / \mathrm{ml}$. The difference in the results might be due to age difference, patients' selection and numbers, and different methods of assessment.

Salivary gland scintigraphy using ${ }^{99 \mathrm{~m}} \mathrm{Tc}$-pertechnetate is the most commonly used radioactive pharmaceutical agent, which roughly correlates with salivary gland biopsy findings, and it is a direct test of secretory function [10]. In this test, the isotope is concentrated 
and excreted by the salivary glands, which allows demonstration of uptake in the salivary glands.

The EF parameter would be most useful to track subtle changes in the salivary gland function, providing a marker for follow-up with time as stated by Loutfi et al. [11].

In our study on comparing EF of salivary glands of the four groups, the SS group was significantly less than those in the other non-SS groups $(P<0.001)$, and this is in accordance with Adams et al. [12]. The level of large salivary glands $\mathrm{EF}$ of up to $33.1 \%$ was determined in our study to identify salivary gland dysfunction with a sensitivity of $100 \%$ and specificity of $86.67 \%$, while in Klutmann et al. [13] the cutoff value for parotid gland EF was less than 28.3\%, and in Schizukuishi et al. [14] it was less than $20.7 \%$ in the submandibular gland.

There was a negative correlation between salivary CXCL13 levels and salivary glands EF $(P<0.001)$, this is in agreement with Nishikawa et al. [15].

Strength of the study: it is the first study to detect scintigraphy in SS in an Egyptian population.

Weak aspects: the small number of patients is a weak point. Future study is recommended on a larger scale of patients.

\section{Conclusion}

Our results recommend the use of salivary CXCL13 as a noninvasive screening tool for the detection of SS.

Larger scale future studies should be done in order to examine the diagnostic as well as the prognostic role of salivary CXCL13 for early detection of SS and for the evaluation of its value in determining the prognosis of this autoimmune disorder.

\section{Declaration of patient consent}

The authors certify that they have obtained all appropriate patient consent forms. In the form the patient(s) has/have given his/her/their consent for his/ her/their images and other clinical information to be reported in the journal. The patients understand that their names and initials will not be published and due efforts will be made to conceal their identity, but anonymity cannot be guaranteed.

\section{Financial support and sponsorship Nil.}

\section{Conflicts of interest}

There are no conflicts of interest.

\section{References}

1 Delaleu N, Madureira AC, Immervoll H, Jonsson R. Inhibition of experimental Sjögren's syndrome through immunization with HSP60 and its peptide amino acids 437-460. Arthritis Rheum 2008; 58:2318-2328.

2 Zhong X, Gao W, Degauque N, Bai C, Lu Y, Kenny J, et al. Reciprocal generation of Th1/Th17 and T(reg) cells by B1 and B2 B cells. Eur J Immunol 2007; 37:2400-2404.

3 Amft N, Bowman SJ. Chemokines and cell trafficking in Sjögren's syndrome. Scand J Immunol 2001; 54:62-69.

4 Kramer JM, Rothstein TL. CXCL13 in Sjogren's syndrome: a novel biomarker of disease. Oral Surg Endodentol 2011; 111:e41.

5 Sherif NM, Arafa MM, Ibrahim SE, Moussa SG. CXC ligand 13 in rheumatoid arthritis and its relation to secondary Sjögren's syndrome. Egypt Rheumatol 2013; 1:121-126.

6 Pedro G, Gintaras J, Maria H, Guobis Z. Diagnostic approaches to Sjögren's syndrome. J Oral Maxillofac Res 2012; 3:e3.

7 Vitali C, Bombardieri S, Jonsson R, Moutsopoulos HM, Alexander EL, Carsons SE, et al. Classification criteria for Sjogren's syndrome: a revised version of the European criteria proposed by the American-European Consensus Group. Ann Rheum Dis 2002; 61:554-558.

8 Kramer JM, Klimatcheva E, Rothstein TL. CXCL13 is elevated in Sjögren's syndrome in mice and humans and is implicated in disease pathogenesis. $J$ Leukoc Biol 2013; 94:1079-1089.

9 Lee KE, Kang JH, Yim YR, Kim JE, Lee JW, Wen L, et al. Predictive significance of CCL21 and CXCL13 levels in the minor salivary glands of patients with Sjögren's syndrome. Clin Exp Rheumatol 2017; 35:234-240.

10 Segovia AD, Ibanez G, Hernandez-Ortiz Cetina JA, Gonzalel-Jimenez Y, Diaz-Jouanen E. Salivary gland involvement in diseases associated with Sjogren's syndrome. 1. Radionuclide and roentgenographic studies. J Rheumatol 1974; 1:159-165.

11 Loutfi I, Nair MK, Ebrahim AK. Salivary gland scintigraphy: the use of semiquantitative analysis for uptake and clearance. J Nucl Med Technol 2003; 31:81-85.

12 Adams BK, Al Attia HM, Parkar S. Salivary gland scintigraphy in Sjögren's syndrome: are quantitative indices the answer? Nucl Med Commun 2003; 24:1011-1016.

13 Klutmann S, Bohuslavizki K, Kröger S, Bleckmann C, Brenner W, Mester J, Clausen M. Quantitative salivary gland scintigraphy. J Nucl Med Technol 1999; 27:20-26.

14 Shizukuishi K, Nagaoka S, Kinno Y, Saito M, Takahashi N, Kawamoto M, et al. Scoring analysis of salivary gland scintigraphy in patients with Sjögren's syndrome. Ann Nucl Med 2003; 17:627-631.

15 Nishikawa A, Suzuki K, Kassai Y, Gotou Y, Takiguchi M, Miyazaki T, et al. Identification of definitive serum biomarkers associated with disease activity in primary Sjögren's syndrome. Arthritis Res Ther 2016; 18:106. 